\title{
POSTURAL CONTROL AND VERTICAL JUMPING PERFORMANCE IN ADOLESCENT AND ADULT MALE BASKETBALL PLAYERS
}

\author{
T. Jallai, J. Ereline, T. Kums, H. Aibast, \\ H. Gapeyeva, M. Pääsuke \\ Institute of Exercise Biology and Physiotherapy, University of Tartu, \\ Tartu, Estonia
}

\begin{abstract}
The purpose of this study was to compare postural control and vertical jumping performance characteristics in adolescent and adult male basketball players. Forty nine male basketball players participated in this study. They were distributed into three groups: (1) young adolescents (YA; with mean age $15.4 \pm 0.7 \mathrm{yrs),} \mathrm{(2)} \mathrm{old} \mathrm{adolescents}$ (OA; 17.9 $\pm 1.2 \mathrm{yrs}$ ), and (3) adults (AD; $25.9 \pm 3.7 \mathrm{yrs).} \mathrm{Static} \mathrm{standing}$ balance during bi- and unipedal standing and vertical jumping characteristics were measured on a force platform. AD basketball players had significantly $(\mathrm{p}<0.05)$ higher vertical jumping height and power development per unit of body mass as compared to YA and OA group; OA group outperformed YA group as well. Static standing stability was better in AD group than YA and OA group. There were no significant differences between $\mathrm{YA}$ and $\mathrm{OA}$ groups in static standing balance test. Age and sports participation were significantly $(p<0.05)$ positively correlated with vertical jumping height and power. Anthropometrical characteristics and sports participation were all significantly $(p<0.05)$ negatively correlated with centre of pressure trace length during bi- and unipedal standing. No significant correlation was observed between balance and vertical jumping characteristics. It was concluded that in male basketball players the improvement in vertical jumping performance and static standing balance during bipedal stance are influenced by sports participation
\end{abstract}


and maturation, whereas static standing balance during unipedal stance is not affected by aforementioned factors.

Key words: postural control, vertical jumping performance, young adults, basketball

\section{INTRODUCTION}

Popular sports, like basketball have high performance demands on an athlete. On the other hand, the risk of sustaining sports injuries is high. Deficits in postural control and muscle power represent two important intrinsic injury risk factors $[3,8,16]$. Age and sports specific training can have an influential effect on these characteristics [5]. Higher-level basketball player is moderately strong, powerful and has a quick, explosive burst of speed, as well as good endurance to repeat it throughout the game $[5,6,12]$. Good flexibility, stability and balance are also required to help reduce the possible incidence of injury [13]. The literature suggests that there is an association between lack of balance $[3,16]$, as well as deficits in muscle strength (lower limb power) and lower extremity injuries [8]. Impaired postural control manifests itself, for example, in prolonged latencies of lower extremity muscles during the compensation of unexpected perturbations. Deficits in muscle strength may cause an imbalance in muscular cocontraction of the ankle or knee joint resulting in reduced joint stiffness during high-load dynamic activities and increase the risk of an injury $[11,13,16]$.

The ability to control the balance of the body while standing is a complex and integrative processing from a variety of sensory and motor inputs [17] and is fundamental for different types of physical activities. It is a basis for further control and coordination of more complex movements and therefore has an important role in sports activities, such as basketball. Although Hobbs [9] concluded that neither dynamic nor static balance is a factor in basketball playing ability, other studies [14] found that performing high-level movements during practice and competition requires both static and dynamic balance. Scores of postural balance are frequently used to identify players with chronic ankle instability [4]. Balance is often measured by having subjects stand on a computerized force-platform. Centre of pressure (COP) motion is indicative of postural control and is 
measured from the ground reaction forces from a force platform. COP displacement is generally considered the gold standard measure of balance. The single-limb stance test on a force-platform is a reliable method [1,2] and is commonly used for evaluation of functional instability and ankle ligament injuries [13, 15, 18]. Cross-sectional studies have found that athletes generally have superior balance ability compared with control subjects [11]. This implies that sport participation improves balance, although it varies between sports. Normally, sports training improves bipedal postural control and decreases the difference between static and dynamic balance. Studies have shown that basketball players have better balance than nonathletes, but worse than soccer players, especially in unipedal balance, because basketball players rarely engage in unilateral stationary balance, whereas soccer players often perform dynamic unilateral movements when kicking the ball [11].

Success in many sports depends heavily upon the athlete's explosive leg power and muscular strength. An athlete must be able to use strength as quickly and forcefully as possible. For the purpose of athletic performance testing for basketball players, the vertical jump test has been shown to be the best predictor of playing time [10]. Variations of vertical jump are the most commonly employed measure for lower body explosive power or speed-strength performance.

The aim of this study was to compare postural control and vertical jumping performance characteristics between adolescent and adult male competitive basketball players.

\section{METHODS}

\section{Subjects}

A total of 49 male basketball players participated in the present study. The subjects were categorized into the following groups: (1) young adolescents (YA) represented players who competed in Estonian B and $\mathrm{C}$ league; (2) old adolescents (OA) represented players who competed in Estonian A league; and (3) adults (AD) represented players who competed in Estonian first league (Table 1). All subjects were confirmed to be healthy and allowed to participate in regular trainings by a medical doctor. Besides, each subject was screened by a questionnaire to exclude those with recent (up to 6 months) ankle sprains or other lower extremity or back complaints. 
Table 1. Age and anthropometric characteristics of the subjects (mean $\pm \mathrm{SD}$ ).

\begin{tabular}{|l|c|c|c|c|c|c|}
\hline Groups & $\mathbf{n}$ & Age (yrs) & $\begin{array}{c}\text { Height } \\
\mathbf{( c m )}\end{array}$ & $\begin{array}{c}\text { Body mass } \\
\mathbf{( k g )}\end{array}$ & $\begin{array}{c}\text { SMI } \\
\left(\mathbf{k g} \cdot \mathbf{m}^{2}\right)\end{array}$ & $\begin{array}{c}\text { Sports } \\
\text { partici- } \\
\text { pation } \\
(\mathbf{y r s})\end{array}$ \\
\hline $\begin{array}{l}\text { Young } \\
\text { Adolescents }\end{array}$ & 26 & $15.4 \pm 0.7$ & $186.2 \pm 8.1$ & $73.9 \pm 9.7$ & $21.3 \pm 2.2$ & $6.4 \pm 2.0$ \\
\hline $\begin{array}{l}\text { Old } \\
\text { Adolescents }\end{array}$ & 16 & $17.9 \pm 1.2$ & $188.3 \pm 4.9$ & $80.8 \pm 10.4$ & $22.7 \pm 2.2$ & $8.9 \pm 1.9$ \\
\hline Adults & 7 & $25.7 \pm 3.7$ & $200.7 \pm 7.0$ & $97.4 \pm 11.3$ & $24.1 \pm 1.9$ & $15.3 \pm 1.9$ \\
\hline
\end{tabular}

BMI - body mass index

\section{Experimental protocol}

The subjects were scheduled for the testing one day after an easy practice or a recovery day. The purpose of the study and procedures were explained to the subjects and all questions were answered. Sex, age, sports participation and lower limb dominance (as determined by asking "Which foot would you use for take-off?") were recorded. After a light warm-up and stretching routine $(10 \mathrm{~min})$ the subjects were measured for body height and weight. The bi- and unipedal static balance testing was performed during standing barefoot on the force platform for $30 \mathrm{~s}$. The subjects were familiarized with the positions before testing.

The vertical jumping tests included 3 sets of squat jumps, countermovement jumps and drop jumps. The best results were used for data analysis. The drop jump box was set at $0.40 \mathrm{~m}$ for optimal drop height. To increase the validity of using vertical jump as a specific measure of lower body power, all vertical jump tests were performed without using the arms (keeping the hands on hips), excluding the contribution of upper limbs to vertical jump movement.

\section{Statistical analysis}

Standard statistical methods were used for the calculation of means, standard error of the means $( \pm \mathrm{SE})$ and standard deviation $( \pm \mathrm{SD})$. A Student unpaired t-test was used to measure differences between groups. Pearson's product-moment correlation was determined between anthropometrical, postural control and vertical jumping characteristics. Statistical significance was accepted at $\mathrm{p}<0.05$. 


\section{RESULTS}

In all three groups there were significant differences $(p<0.05)$ in age, body mass and sports participation in years (Table 1). AD group was significantly different $(\mathrm{p}<0.05)$ from YA and OA group in height. YA group was significantly different $(\mathrm{p}<0.05)$ from $\mathrm{OA}$ and $\mathrm{AD}$ group in BMI.

AD group had significantly higher vertical jumping height (Figure 1) and power per unit of body mass (Figure 2) as compared to YA and OA group; OA group outperformed YA group as well (Figures 1 and $2)$. In balance tests $A D$ group had significantly lower COP trace length in bipedal stance than YA group and OA (Figure 3). There were no significant differences $(\mathrm{p}>0.05)$ between YA and OA group in balance tests (Figures 3 and 4).

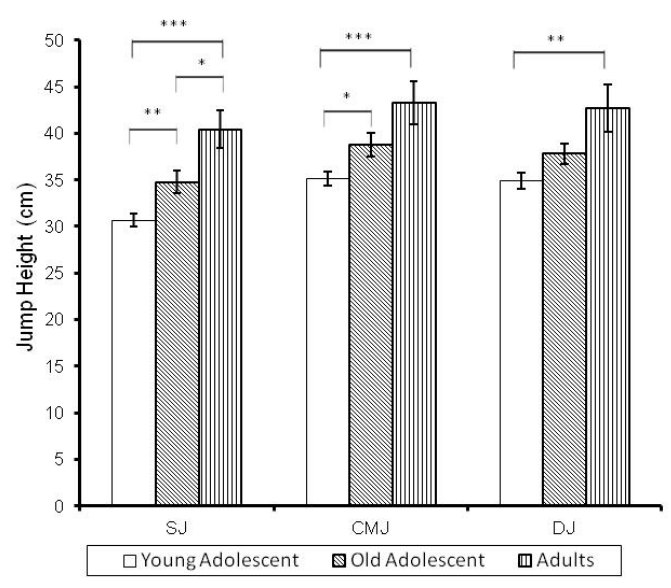

Figure 1. Vertical jumping height in squat jump (SJ), counter movement jump (CMJ) and drop jump (DJ) in male basketball players (mean \pm SE). * $\mathrm{p}<0.05 ; * * \mathrm{p}<0.01 ; * * * \mathrm{p}<0.001$. 


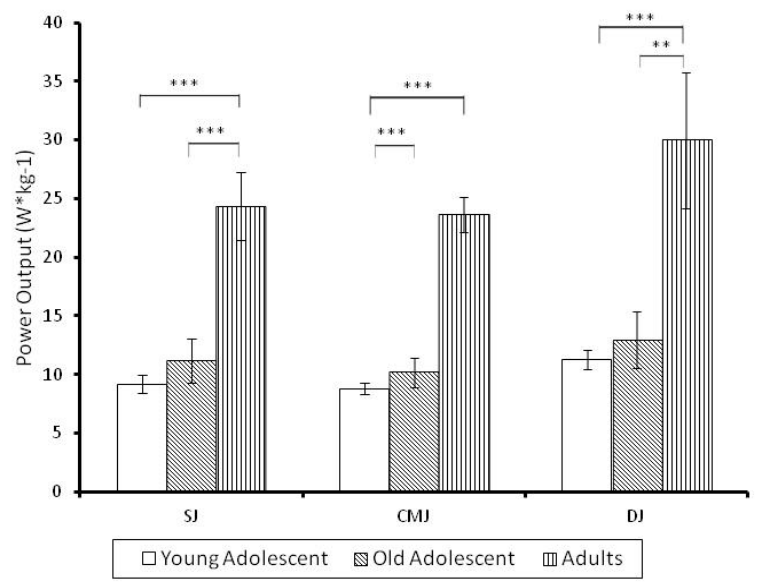

Figure 2. Power output per unit of body mass in squat jump (SJ), counter movement jump (CMJ) and drop jump (DJ) in male basketball players (mean $\pm \mathrm{SE}$ ). ${ }^{* *} \mathrm{p}<0.01 ; * * * \mathrm{p}<0.001$

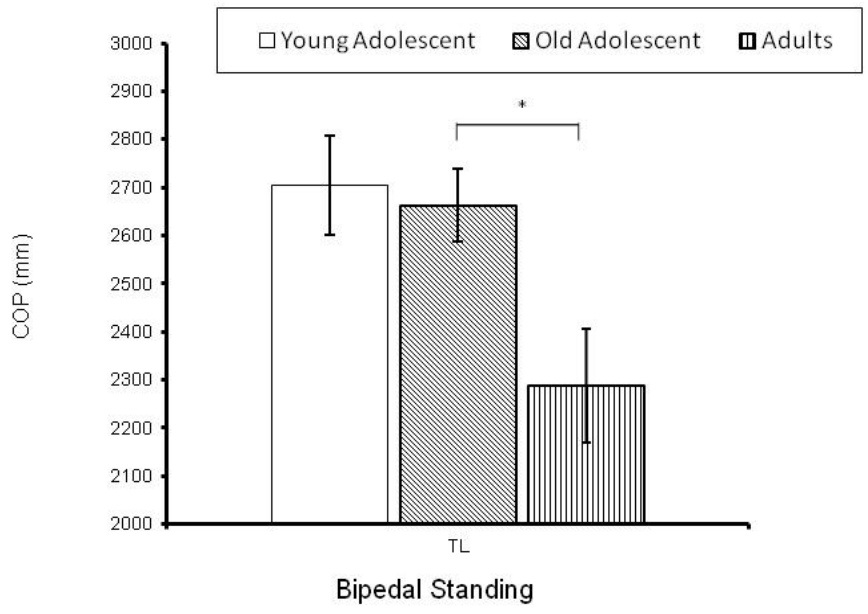

Figure 3. Center of pressure (COP) trace length (TL) during bipedal stance during $30 \mathrm{~s}$ standing test (mean $\pm \mathrm{SE}) .{ }^{*} \mathrm{p}<0.05$. 


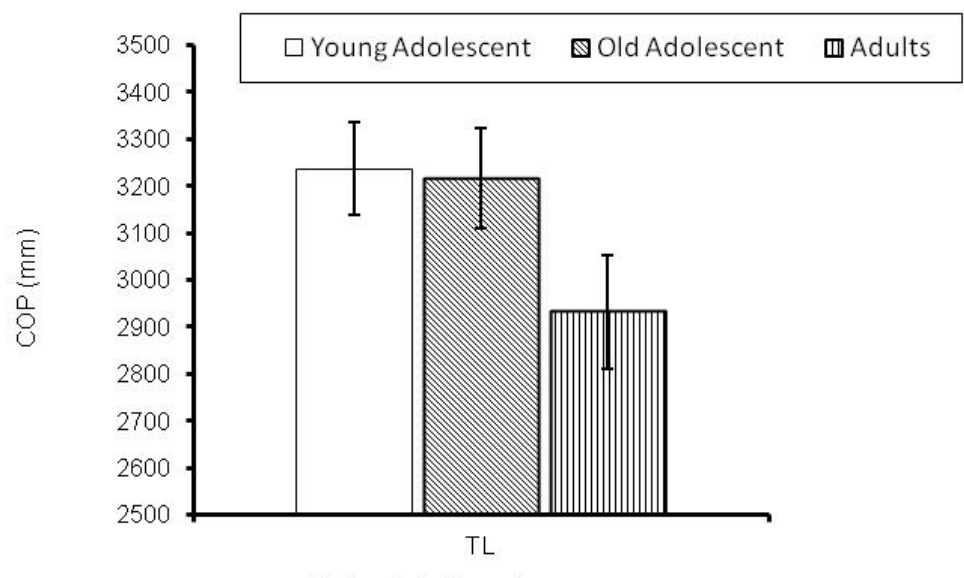

Unipedal Standing

Figure 4. Center of pressure (COP) trace length (TL) during unipedal stance during $30 \mathrm{~s}$ standing test (mean $\pm \mathrm{SE}$ ).

Age and sports participation had medium to high correlations with vertical jumping performance characteristics $(r=0.48$ to $0.81, p<0.05)$ in different measured groups. Body mass and BMI were negatively correlated with vertical jumping height in all groups, having significant correlation in OA group ( $\mathrm{r}=0.58$ to $0.59, \mathrm{p}<0.05$ ). Age, sports participation and anthropometrical characteristics were all negatively correlated with bi- and unipedal COP trace length, but only anthropometrical characteristics had significant correlation $(\mathrm{r}=-0.46$ to $-0.80, \mathrm{p}<0.05)$. No significant correlation was observed between balance and vertical jumping characteristics.

\section{DISCUSSION}

This study compared postural control and vertical jumping performance in three groups of male basketball players. Rates of growth and maturation can vary widely within a group of people of same chronological age. The difference of maturation in males in young and teenage groups and difference of anthropometrical characteristics of different playing positions in adolescent group made the groups less homogenous. Besides, basketball players have numerous smaller or 
bigger injuries throughout their athletic career, which they often do not mention. Thus, the interpretation of results, especially in balance testing, is complicated.

Age, height, body mass, BMI and sports participation correlated with vertical jumping and balance characteristics. Body mass and BMI had positive correlations with relative power in young adolescent and old adolescent groups, whereas these characteristics had negative correlations with relative power in adult group. This suggests that it is important to take into consideration body composition - "active and passive" body mass. Body mass and BMI had clearly negative effect on vertical jumping height in all groups. Negative correlation existed between balance and anthropometrical characteristics, as well as age and sports participation (number of years in basketball practice). Maturation and basketball specific practice improved jumping performance and balance, although no significant correlation existed between age, sports participation and balance. This implies that complimentary to basketball practice, a specific balance and coordination training can be beneficial. Static balance testing alone is probably not sufficient to state this as a proven conclusion. For future research, dynamic balance testing and body composition should be taken into consideration.

Squat jump is a standard test for concentric power production [5]. It is performed from a set squat depth without any countermovement. Countermovement jump is a standard test for combined concentric and stretch-shortening cycle (SSC) performance [5]. Drop jump test is used to assess lower limb reactive (speed-) strength and fast SSC performance [5]. Vertical jumping performance is affected by factors that are trainable and can be manipulated by the athlete (muscular power, coordination of muscles, body weight and composition) and features that cannot be influenced by the athlete (genetic make-up, muscle fibre type, central nervous system efficiency). High efficiency of vertical jump movement allows an athlete to generate the most amount of power with the least amount of effort. Basically the leaner the athletes are (low in body fat) and the less unnecessary muscle mass they have, the higher they are able to jump. Less weight means there is less power needed to jump the same height. Reactive strength is more impacted by body weight, including excess muscle mass. The speed of strength application is often broadly referred to as rate of force development and is an important attribute for vertical jumping performance [7]. 
In conclusion, the present study indicated that in male basketball players the improvements in vertical jumping performance and static standing balance during bipedal stance are influenced by sports participation and maturation, whereas static standing balance during unipedal stance is not affected by aforementioned factors.

\section{REFERENCES}

1. Ageberg E., Zatterstrom R., Moritz U. (1998) Stabilometry and oneleg hop test have high test-retest reliability. Scand. J. Med. Sci. Sports. 8: 198-202

2. Ageberg E., Roberts D., Holmstrom E., Friden T. (2003) Balance in single-limb stance in healthy subjects - reliability of testing procedure and the effect of short-duration sub-maximal cycling. BMC Musculoskel. Disord. 4: 14-30

3. Alexander K. M., LaPier T. L. K. (1998) Differences in static balance and weight distribution between normal subjects and subjects with chronic unilateral low back pain. J. Orthop. Sport Phys. Ther. 28: 378-383

4. Brown, C. N. and Mynark R. (2007). Balance deficits in recreational athletes with chronic ankle instability. J. Athl. Training. 42: 367-373

5. Gamble P. (2010). Strength and conditioning for team sports. Sportspecific physical preparation for high performance. Routledge, London and New York

6. Gillam, G. M. (1985) Identification of anthropometric and physiological characteristics relative to participation in college basketball. Nat. Strength Cond. Assoc. J. 7: 34-36

7. Gruber M., Gruber S. B. H., Wolfgang T., Schubert M., Beck S. C., Gollhofer A. (2007) Differential effects of ballistic versus sensorimotor training on rate of force development and neural activation in humans. J. Strength Cond. Res. 21: 274-282

8. Hakkinen K. et al. (2003) Neuromuscular adaptations during concurrent strength and endurance training versus strength training. Eur. J. Appl. Physiol. 89: 42-52

9. Hobbs M. L. (2008). Dynamic balance and basketball playing ability. Theses and Dissertations-Health and Human Performance. http://ecommons.txstate.edu/hpertad/3

10. Hoffman J. R., Tenenbaum G., Maresh C. M. and Kraemer W. J. (1996) Relationship between athletic performance tests and playing time in elite college basketball players, J. Strength Cond. Res. 10: $67-71$ 
11. Hrysomallis C. (2011). Balance ability and athletic performance. Sports Med. 41: 221-232

12. Latin R. W., Berg K., and Baechle T. (1994) Physical and performance characteristics of NCAA Division I male basketball players. J. Strength Cond. Res. 8: 214-218

13. Leanderson J., Wykman A., Eriksson E. (1993) Ankle sprain and postural sway in basketball players. Knee Surg. Sports Trauma. Arthrosc. 1: 203-205

14. Perrin P., Deviterne D., Hugel F., Perrot C. (2002) Judo, better than dance, develops sensorimotor adaptabilities involved in balance control. Gait Posture. 15: 187-194

15. Rogind H., Lykkegaard J. J., Bliddal H., Danneskiold-Samsoe B. (2003) Postural sway in normal subjects aged 20-70 years. Clin. Physiol. Funct. Imag. 23: 171-176

16. Surve I., Schwellnus M. P., Noakes T., Lombard C. (1994) A fivefold reduction in the incidence of recurrent ankle sprains in soccer players using the sport-stirruporthosis. Am. J. Sports Med. 22: 601-606

17. Teasdale N., Stelmach G. E. and Breunig A. (1991) Postural sway characteristics of the elderly under normal and altered visual and support surface conditions. J. Geronthol. 46: 238-244

18. Tropp H., Odenrick P. (1988) Postural control in single-limb stance. J. Orthop. Res. 6: 833-839

\section{Correspondence to:}

Tarmo Jallai

Institute of Exercise Biology and Physiotherapy,

University of Tartu, Estonia

Jakobi Street 5, 51014, Tartu

Estonia

E-mail: tarmoj@ut.ee 